\title{
Serotonin regulates hepcidin expression via a gut-liver axis
}

T. Coman ${ }^{1,2 *}$, M. Falabrègue $2,3,4,5$, J. Rossignol ${ }^{2}$, P. Bonneau ${ }^{2}$, M. Djebar ${ }^{2}$, A. Bigorgne $^{2}$, J R R. Mathieu ${ }^{5,6}$, M. Foretz ${ }^{6}$, H. Puy ${ }^{4,7}$, K. Peoc'h ${ }^{4,7}$, D. Logeart ${ }^{8}$, A. Cohen-Solal ${ }^{8}$, J.-M. Launay ${ }^{8}$, L. Maroteaux ${ }^{9}$, C. Peyssonnaux ${ }^{5,6}$, O. Hermine ${ }^{2,4,5,10}$, F. Côtéé, $, 4,5,6 *$

1 Institut Gustave Roussy, Villejuif, France.

2Institut Imagine, INSERM U1163, Paris, France.

${ }^{3}$ Institute for Research in Biomedicine and Epidemiology of Sport (IRMES), EA 7329, Paris, France.

${ }^{4}$ Université de Paris, Paris, France.

${ }^{5}$ Laboratoire d'Excellence GR-Ex, Paris, France.

6Institut Cochin, INSERM U1016, CNRS UMR 8104, Paris, France.

${ }^{7}$ INSERM UMR-S 1149, CRI, UFR de Médecine Xavier Bichat, Paris, France.

8 INSERM UMR-S 942, Lariboisière Hospital, Paris, France.

9 Institut du Fer à Moulin, Sorbonne University, Paris, France.

${ }^{10}$ Department of Hematology, Necker Hospital, Assistance Publique des Hôpitaux de Paris, Paris, France.

*Corresponding author. francine.cote@inserm.fr

\begin{abstract}
Liver hepcidin, is well recognized as the central hormone of systemic iron regulation. Although serotonin is most recognized as a brain neurotransmitter, prodigious quantities are synthesized in gut enterochromaffin cells and several lines of evidence, also identified the gut as an essential sensor and regulator of iron homeostasis. Using a mouse model deficient for peripheral serotonin (Tph1 KO), we identified gut-derived serotonin as a key physiological factor in hepcidin regulation. Serotonin represses hepcidin's through a $5 \mathrm{HT} 2 \mathrm{~B}$ receptor-dependent pathway, independently of any other known hepcidin regulators, including bone marrow signals. This regulation is conserved in humans and shows physiological significance as a negative correlation between serotonin and hepcidin levels was observed in a cohort of healthy individuals. Moreover, in pathological situation such as acute heart failure, where iron deficiency has a negative prognostic impact, we provide evidence that an increase in serotonin level seems necessary to repress hepcidin level, to increase iron availability.
\end{abstract}

\section{Keywords}

Serotonin, iron, hepcidin, gut, hypoxia 


\section{Introduction}

Iron is essential to vital biological processes in all living organisms. Despite years of public health efforts and intense research, iron imbalance still concerns over $25 \%$ of the world's population and is linked to numerous comorbidities associated with poorer prognoses and higher treatment costs.

Hepcidin is the central hormone regulating systemic iron homeostasis. Highly conserved between species, hepcidin lowers plasma iron concentrations by binding to ferroportin (SLC40A1), the only known iron exporter, which leads to iron retention in macrophages and enterocytes (Nemeth et al., 2004). Because of the central role hepcidin plays in systemic iron homeostasis, there have been extensive efforts to identify factors regulating its expression (Roth et al., 2019). TFR1 signaling, due to iron excess, and IL-6 signaling, associated with chronic or acute inflammation, stimulates hepcidin production. In contrast, hepcidin is repressed by iron deficiency, erythroid expansion, and hypoxia. There is evidence that, in murine models, erythroferrone (ERFE) released by erythroid progenitors in response to acute EPO stimulation acts as an early regulator to increase iron availability during erythroid expansion (Kautz et al., 2014). However, increasing evidences suggest that hepcidin regulation occurs independently of any erythropoietic signal in the bone marrow (BM).

The gut has a unique physiological hypoxia profile (Shepherd, 1982)(Taylor and Colgan, 2007). Enterochromaffin cells, the most abundant type of enteroendocrine cells, express HIF-1 $\alpha$, the canonical regulator of hypoxic responses, and have shown to be sensors of fluctuation in an oxygen environment (Haugen et al., 2012). Furthermore, the main secretory product of enterochromaffin cells is serotonin (5$\mathrm{HT}$ ), which has been extensively studied as a neurotransmitter. Yet $>10$ times more $5-\mathrm{HT}$ is produced in the gut than in the central nervous system (CNS), and gut $5-\mathrm{HT}$ accounts for $90 \%$ of peripheral $5-\mathrm{HT}$ (Walther et al., 2003)(Côté et al., 2003). Under hypoxic conditions, the enzyme TPH1, responsible for 5-HT synthesis, is strongly upregulated, suggesting that $\mathrm{HIF}-1$ a may directly drive $5-\mathrm{HT}$ production in cells subject to hypoxia (Pocock and Hobert, 2010). Previous studies indicated that enterochromaffin derived 5-HT stimulates gut contractility. Yet mice deficient for peripheral 5-HT (Tph1 KO) do not experience any difference in gut contractility (Gershon, 2012), which leaves the role of the huge quantities of 5-HT synthesized in enterochromaffin cells unexplained.

Here we show that peripheral 5-HT produced by the gut in response to physiological or acute hypoxia regulates hepcidin expression in the liver, independently of any BM erythropoietic activity. 


\section{Results and discussion}

We previously showed that 5-HT deficient mice (Tph1 KO) presented a phenotype of ineffective erythropoiesis and that $5-\mathrm{HT}$ produced by proerythroblasts was necessary for normal erythropoiesis and red blood cells survival (Amireault et al., 2011)(Amireault et al., 2013). In Tph1 KO mice, the dysplastic BM phenotype was associated with an excess of BM sideroblasts, reminiscent of what is seen in patients with refractory anemia with ring sideroblasts (RARS), often associated with iron overload (Sibon et al., 2019). However, in Tph1 KO mice, serum iron and transferrin concentrations were in the normal range (Fig. 1, A and $B$ ), and ferritin concentration, though significantly higher, was still abnormally low in Tph1 KO mice in light of what one would expect with RARS (Fig. 1C). When investigating hepcidin concentrations, we observed a significant increase in steady-state Hamp1 expression in Tph1 KO mice's livers, contrary to what is expected for RARS (Fig. 1D). Hepcidin increased in Tph1 KO mice despite high plasma EPO concentrations (Amireault et al., 2011) and no inflammation signs (Fig. 1E). These results led us to hypothesize that peripheral 5-HT inhibits hepcidin expression physiologically.

To test this hypothesis, we first investigated whether 5-HT could directly regulate hepcidin expression in vitro. Wild type (WT) murine primary hepatocyte cell cultures were treated with 5-HT or BW723C86, a 5$\mathrm{HT}_{2 \mathrm{~A} / 2 \mathrm{~B}}$ receptor agonist. Hamp1 mRNA levels decreased by almost $60 \%$ as early as $4 \mathrm{hr}$ following either treatment $($ Fig. $1 \mathrm{~F})$. We previously demonstrated the $5-\mathrm{HT}_{2 \mathrm{~B}}$ receptor $\left(5-\mathrm{HT}_{2 \mathrm{~B}} \mathrm{R}\right)$ presence on hepatocytes (Ebrahimkhani et al., 2011). To further confirm the involvement of $5-\mathrm{HT}_{2 \mathrm{~B}} \mathrm{R}$ signaling in hepcidin repression, we measured ex vivo Hamp1 expression in livers of mice deficient in $5-\mathrm{HT}_{2 \mathrm{~A}} \mathrm{R}, 5-\mathrm{HT} \mathrm{T}_{2 \mathrm{~B}} \mathrm{R}, 5-\mathrm{HT}_{2 \mathrm{~A} / 2 \mathrm{~B}} \mathrm{R}$, or the serotonin specific transporter (SERT). Hamp1 expression in mice lacking $5-\mathrm{HT}_{2 \mathrm{~B}} \mathrm{R}$ or $5-\mathrm{HT}_{2 \mathrm{~A} / 2 \mathrm{~B}}$, as in Tph1 $\mathrm{KO}$ mice, was significantly higher than in those deficient in $5-\mathrm{HT}_{2 \mathrm{~A}} \mathrm{R}$ and SERT, which were similar to WT animals (Fig. 1G).

We then investigated the mechanism through which $5-\mathrm{HT}$ could repress Hamp1 expression. It is well established that BMP signaling—especially involving BMP6, which activates the $\mathrm{Smad}_{1 / 5 / 8}$ pathway through interaction with its cognate receptor-induces hepcidin production (Meynard et al., 2009). As 5-HT inhibition of BMP signaling has previously been demonstrated (Long et al., 2006), we used immunofluorescence to measure steady-state $\mathrm{Smad}_{1 / 5 / 8}$ protein expression in liver sections from Tph1 KO, and WT mice. In Tph1 $\mathrm{KO}$ mice, the $\mathrm{pSmad}_{1 / 5 / 8} / \mathrm{Smad}_{1 / 5 / 8}$ ratio was significantly higher in the nucleus than in the cytoplasm (Fig. 1, $\mathrm{H}$ to J). This observation suggests abnormal activation of the BMP signaling pathway in the absence of 5 $\mathrm{HT}$. Together, these data indicate that a $5-\mathrm{HT}$ signal via the $5 \mathrm{HT}_{2 \mathrm{~B}}$ receptor controls hepcidin expression by modulating BMP/Smad-induced Hamp1 activation. 
A

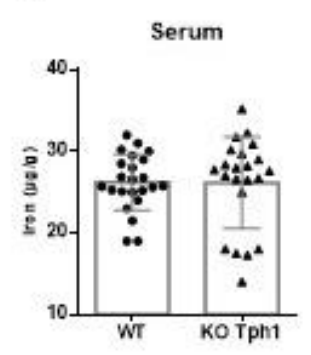

$E$
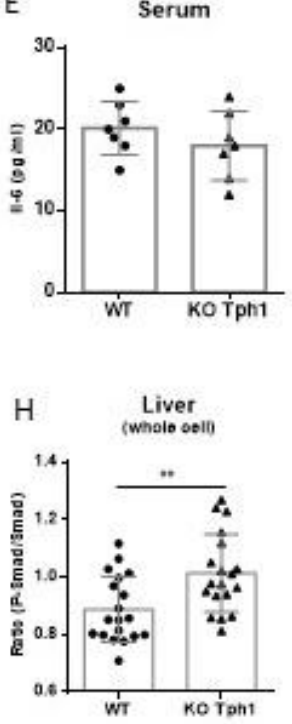

B

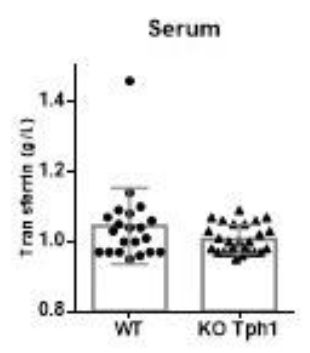

$\mathrm{C}$

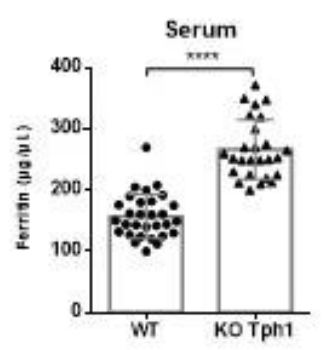

D

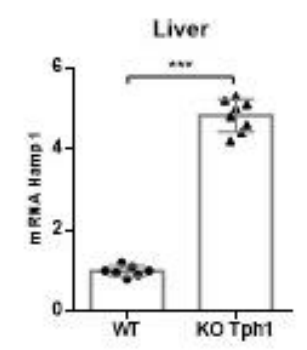

$\mathrm{F}$

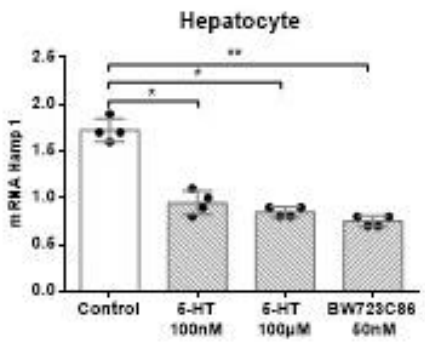

G

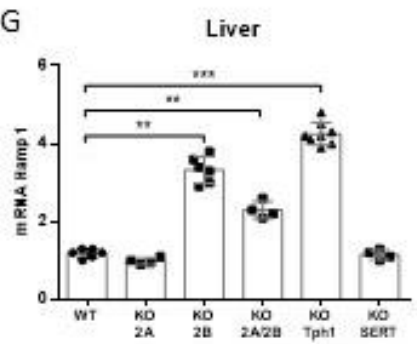

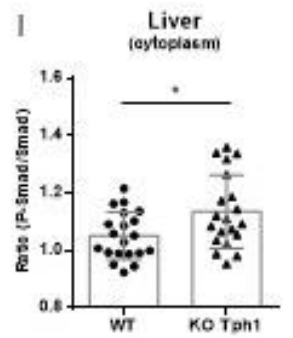

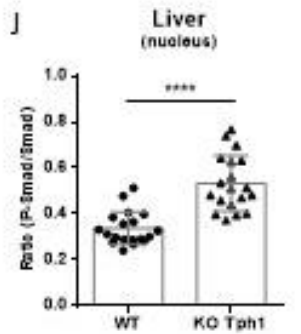

Fig. 1. Hepcidin concentration increased in mice deficient for peripheral serotonin, independently of known hepcidin regulators, and can repress Hamp1 expression in hepatocytes through $5-\mathrm{HT}_{2 \mathrm{~B}}$ receptor signaling. Serum (A) iron, (B) transferrin, and (C) ferritin concentrations; (D) liver Hamp1 expression as evaluated by qPCR analysis; and (E) serum IL-6, in Tph1 KO $(n=6-26)$ versus WT( $n=7-30)$, miœ. (F) Hamp1 expression (qPCR) in primary murine hepatocytes cultured in vitro with 5-HT or BW723C86 (Data are from 2 independent experiments). (G) Comparison of Hamp1 expression (qPCR) in mice KO for Tph1, 5- $\mathrm{HT}_{2 \mathrm{~A}}, 5-\mathrm{HT}_{2 \mathrm{~B}}, 5-\mathrm{HT}_{2 \mathrm{~N} 2 \mathrm{~B}}$, and SERT, and in WT miœ (WT $n=8)$, Tph1 KO $n=8), 5-H T 2 A R ~ K O ~(n=4), 5-H T 2 B R$ KO $(n=6)$, SERT KO $(n=4), 5$ HT2A2BR KO $(n=4)$. Ratio of P-Smad ${ }_{150}$ to $\mathrm{Smad}_{15 / 2 \mathrm{a}}$ in $(\mathrm{H})$ whole cells, (I) cytoplasm, $(\mathrm{J})$ and nucleus, as evaluated by immunofluoresœnce in liver sections. The qPCR analyses of Hamp1 expression were nomalized with beta-actin.

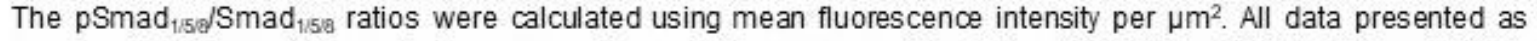
mean $\pm \mathrm{SD}$. Mann-Whitney tests were performed where appropriate. ${ }^{\star} P<0.05,{ }^{\star \star} P<0.005$, ${ }^{\star \star \star} P<0.0005$, $\star \star \star \star x<0.0001$.

As (i) enterochromaffin cells are the primary source of peripheral 5-HT, (ii) hypoxia-responsive elements (HREs) were identified in the Tph1 promoter region (Haugen et al., 2012) (Sibon et al., 2019) and (iii) 5-HT production and secretion are known to be upregulated through a HIF1a-sensitive mechanism in $C$. elegans (Pocock and Hobert, 2010), we hypothesized that Tph1 and 5-HT synthesis induced by hypoxia in enterochromaffin cells could repress Hamp1 in hepatocytes. In support of this hypothesis, a significant increase in Tph1 mRNA expression and 5-HT synthesis was explicitly observed in the duodenum, not the colon, of a mouse model of prolonged hypoxia, after 6hr (Fig. 2, A and B). Additionally, 5-HT was not metabolized at its production site, as shown by low concentrations of its 5HIAA metabolite (Fig. 2C). There 
was a significant increase in the concentration of 5-HT in the portal vein (Fig. 2D). The portal vein directly connects the gut and the liver, suggesting that a high amount of 5-HT is available for direct signaling on hepatocytes. Studies by Choi et al. (Choi et al., 2018) and others have shown that 5-HT portal blood concentrations may reach levels high enough to have endocrine effects on the liver (Young et al., 2018), possibly through hepatocytes' direct signaling.

We previously demonstrated that 5-HT could be produced in BM proerythroblasts (Sibon et al., 2019) and given that erythropoiesis plays a role in Hamp1 regulation (through ERFE production), we next investigated whether medullary 5-HT might also be involved in Hamp1 regulation. After irradiation, Tph1 KO mice were transplanted with WT BM, while WT mice were transplanted with Tph1 KO BM and then exposed to hypoxia. Tph1 mRNA expression increased after 6hr of hypoxia in WT duodenum, regardless of whether the BM was WT or Tph1 KO (Fig. 2E), providing further evidence of the duodenal role in Hamp1 regulation suggested by Fig. 2A. Indeed, in contrast with WT gut mice, Tph1 KO gut mice were unable to repress Hamp1 expression (Fig. 2F). Furthermore, following $6 \mathrm{hr}$ of hypoxia, WT gut/Tph1 KO BM mice could significantly repress Hamp1 (by 59\%). In contrast, Tph1 KO gut/WT BM mice (those incapable of gut 5-HT synthesis) failed to do so. We did, however, observe a slight decrease (21\%), albeit not significant, in hepcidin level in Tph1 KO gut/WT BM mice, which might be the result of a signal from the BM (ERFE or BMderived 5-HT) exerting a synergistic or time-dependent effect alongside that of gut-derived 5-HT. While ERFE levels have been shown to increase in response to EPO after acute anemia, mice lacking ERFE have normal hepcidin concentrations at baseline, suggesting that ERFE is a stress-induced, not a steady-state, regulator. In contrast, 5-HT appears to be a permanent regulator with a gut response to hypoxia, independently of anemia/EPO or any BM erythroid factor.
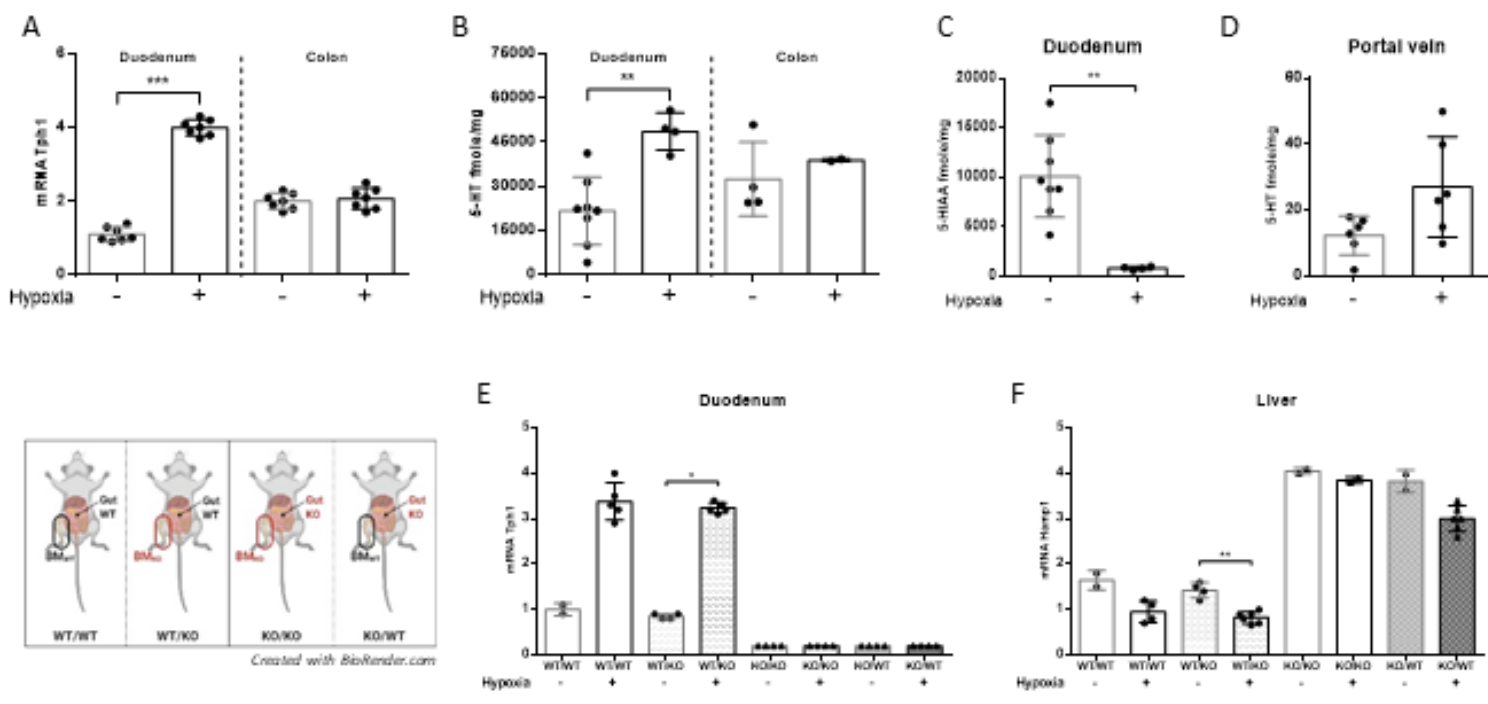

Fig. 2. Duodenum and not bone marrow derived serotonin is responsible for hepcidin regulation (A) Duodenal and colonic Tph1 expression (qPCR) ( $\mathrm{n}=7$ Tph1 mice +l-hypoxia), (B) duodenal and colonic 5-HT levels, (C) duodenal 5-HIAA levels, and (D) portal vein 5-HT levels in WT mice exposed to normoxia or hypoxia. Bone marrow (BM) transplant experiments showing relative expression of $(E) T p h 1$ in the duodenum and $(F)$ Hamp1 in the liver of transplanted mice exposed to nomoxia or hypoxia. WTWT: WT gut, WT BM; WT/KO: WT gut, Tph1 KO BM; KO/KO: Tph1 KO gut / Tph1 KO BM; KO/WT: Tph1 KO gut, WT BM). The qPCR analyses of Hamp1 and Tph1 expression were nomalized with beta-actin. All data presented as mean $\pm \mathrm{SD}$. Mann-Whitney tests were performed where appropriate. ${ }^{\star} P<0.05,{ }^{\star \star} P<0.005,{ }^{\star \star *} P<0.0005$. 
We next hypothesized that $5-\mathrm{HT}$ could also regulate hepcidin expression in human cells. As early as 3 hr after HepG2 cells were subjected to $5-\mathrm{HT}$ or a 5-HT agonist, Hamp1 expression dropped by more than $60 \%$ (Fig. 3A). We further measured hepcidin and 5-HT concentrations in 109 healthy individuals and revealed a significant negative correlation $(P=0.0041)$ between $5-\mathrm{HT}$ and circulating hepcidin concentrations without a sign of inflammation (normal CRP concentrations; data not shown) (Fig. 3B).

While 5-HT appears to be a physiological regulator of hepcidin, we ask whether this regulation could also be relevant in pathological conditions associated with hypoxia. Chronic heart failure (CHF) is a disease where hypoxia is a common symptom during acute decompensation and where iron deficiency was demonstrated to have a negative prognostic impact (Anker et al., 2009). We retrospectively analyzed a cohort of 45 patients with acutely decompensated CHF (Van Aelst et al., 2017) and measured circulating hepcidin, 5-HT and venous lactates at the beginning (day 0) and after resolution of the episode (day 30). As expected in CHF, lactates level was significantly higher on day 0 but similar to controls on day 30 (fig. $3 \mathrm{C}$ ), suggesting that a tissue hypoxia occurred on day 0 . In patients, iron deficiency was reported on day 0 , and iron levels were resolved in most patients on day 30 (Van Aelst et al., 2017). On day 0, we observed a significant correlation between 5-HT and lactate levels (Fig. 3D) implying an increase in 5-HT levels after hypoxia. At this same time point, there was a significant correlation between hepcidin and 5-HT levels (Fig. $3 \mathrm{E}$ ), whereas this correlation disappeared on day 30; as lactates level returned to normal (Fig. 3F). Precisely when we compare patients with high vs low lactate, in high lactate conditions, the level is significantly reduced (Fig. 3E) on day 30 concomitantly with a robust reduction in 5-HT levels (Fig. 3F) and a significant increase in hepcidin levels (Fig. 3G). Altogether, the results indicate that during acute hypoxia, as observed in acute heart failure, an increase in $5-\mathrm{HT}$ level is necessary to repress hepcidin level leading to increase iron bioavailability. Moreover, iron deficiency during acute heart failure might reflect a failure of the $5-\mathrm{HT}$ system to respond to hypoxia.

Overall, the genetic and biochemical evidences presented here reveal the importance of $5-\mathrm{HT}$ as a gut signal triggered by hypoxia, to regulate hepcidin expression, independently of any BM-derived signals. Our findings further imply that the 5-HT regulation of hepcidin is a physiological process with pathological applications. 
Fig. 3. Significant correlation between serotonin and hepcidin levels in individuals under steady state and during acute hypoxia.

(A) qPCR analysis measuring Hamp1 expression in HepG2 cell line with 5 $\mathrm{HT}$ or 5 -HT agonist (DOI). All qPCR analysis for Hamp1 was normalized to beta-actin expression (Data are pooled from 2 independent experiments). (B) Negative correlation between blood $5-\mathrm{HT}$ and hepcidin in blood sample from healthy humans $(n=109)$. (C) Venous lactate measurements $(\mathrm{nM})$ in healthy individuals $(n=109)$ compared to patient with a chronic heart failure (CHF) at day 0 and 30 days after $\mathrm{CHF}$ episode $(n=45)$. Red symbol represent patients with high lactate level. (D) Significant positive correlation between blood $5-\mathrm{HT}$ and venous lactate from $\mathrm{CHF}$ patients at day 0 $(n=45)$. (E) Variation in lactate level $(\mathrm{mM})$ between day 0 and day 30 (recovery) from CHF patients with low lactate level at day 0 (black, $n=28$ ) and CHF patients with high lactate level at day0 (red $n=17$ ). (F) Variation in blood 5-HT level (nM) between day 0 and day 30 from $\mathrm{CHF}$ patients with low lactate level at day 0 (black, $n=28$ ) and CHF patients with high lactate level at day 0 (red $n=17$ ) (G) Variation in hepcidin level $(\mu \mathrm{g} / \mathrm{L})$ between day 0 and day 30 from CHF patients with low lactate level at day 0 (black $n=28$ ) and CHF patients with high lactate level at day 0 (red $n=17$ ). $A$ to $D$, Throughout data are mean \pm SD calculated using an unpaired Mann-Whitney t-test when appropriate or Pearson correlation. E to $G$, Throughout data are mean \pm SD calculated using a paired-t test; ${ }^{* *} p<$ 0.05 and ${ }^{\star \star * \star} p<0.0001$.
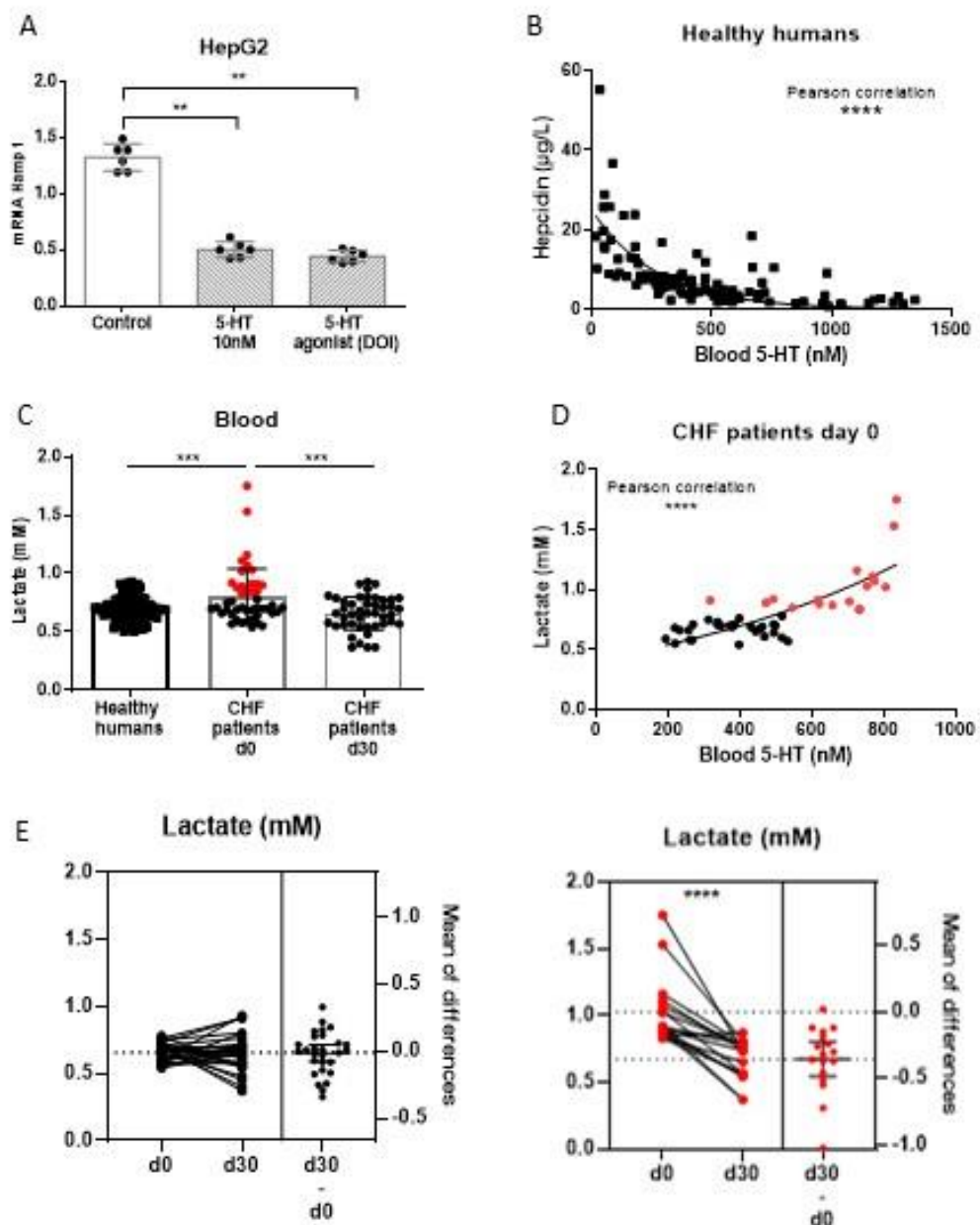

$F$

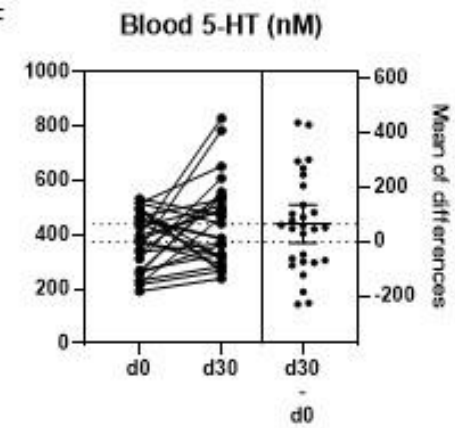

G

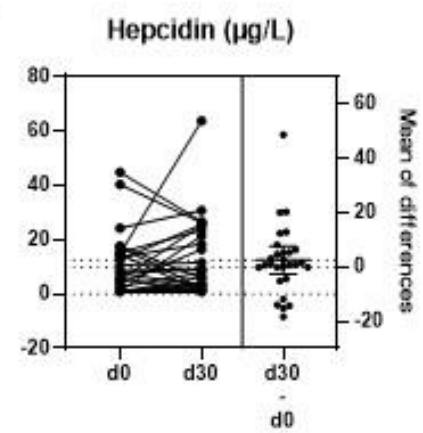

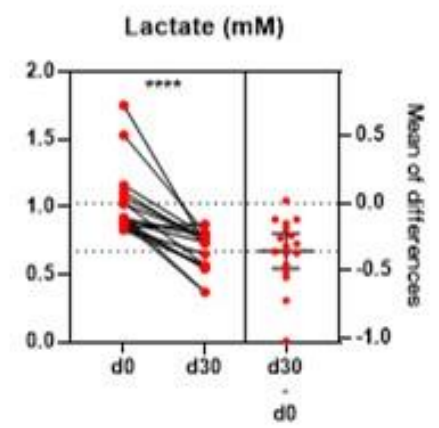

Blood 5-HT (nM)

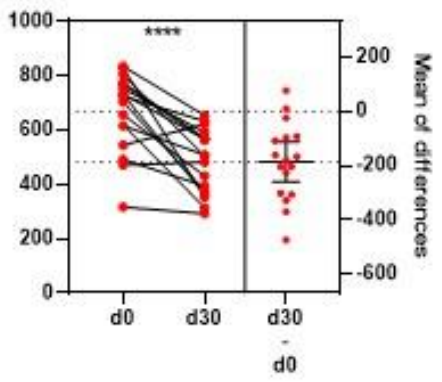

Hepcidin ( $\mu g / L)$

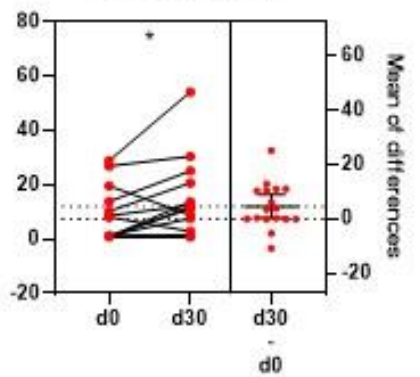




\title{
Acknowledgements:
}

The authors are grateful to Sophie Vaulont for scientific advice and critical reading of the manuscript. The authors thank Aurélia Dujardins and the LEAT Animal Facility for care and breeding of the mice. The authors would also like to thank Jason Miller for editing of the manuscript.

\section{Funding:}

This work was supported in part by grants from Laboratory of Excellence GR-Ex, reference ANR-11-LABX0051. The labex GR-Ex is funded by the program "Investissements d'Avenir" of the French National Research Agency, reference ANR-11-IDEX-0005-02. TC was supported by a Ph.D. fellowship from Institut Imagine. MFa was supported by a Ph.D. fellowhip form Labex Grex. JR was supported by a Ph.D. fellowship from the Ministère Supérieur de l'Enseignement et de la Recherche.

\author{
Author contributions: \\ Design and analysis of the experiments: TC, MFa, JR, FC \\ Performed the experiments: TC, MFa, JR, PB, MD, AB, KP, JML, ACS, DG, HP, FC \\ Technical support and mice models: JRRM, MFo, LM \\ Funding acquisition: $\mathrm{FC}, \mathrm{OH}$ \\ Project administration: FC \\ Supervision: $\mathrm{FC}, \mathrm{CP}, \mathrm{OH}$ \\ Writing - original draft: TC, MFa, CP, FC
}

\section{Competing interests:}

Authors declare that they have no competing interests

\section{Data and materials availability}

The datasets generated during and/or analyzed during the current study are available from the corresponding author on reasonable request. 


\section{Materials and Methods \\ Animal procedures}

Tph1 KO mice were generated as described by Côté et al. (Côté et al., 2003). Tph1 KO and WT animals were derived from pure $\mathrm{C} 57 \mathrm{BL} / 6 / \mathrm{J}(\mathrm{H} 2 \mathrm{~b})$ genetic backgrounds. For some experiments, $\mathrm{C} 57 \mathrm{BL} / 6 / \mathrm{J}$ mice were purchased from Janvier Labs. 5-HT2A, 5HT2B KO, and SERT KO mice were kindly provided by L. Maroteaux. All mice were bred and housed in a pathogen-free facility, within microisolator cages, and used at 8 to 12 weeks of age. For BM transfer experiments Tph1 KO and WT recipients were administered 600 -cGy total body X-ray irradiation on day -1 . This was followed by intravenous infusion into the caudal vein of Tph1 KO or WT donor BM cells. Different groups (for each, $n=12$ ) were constituted: WTMT: WT gut, WT BM; WT/KO: WT gut, Tph1 KO BM; KO/KO: Tph1 KO gut / Tph1 KO BM; KO/WT: Tph1 KO gut, WT BM. Animal experiments were performed according to the recommendations of the ethical review board. For hypoxia experiments, WT and Tph1 KO mice were transferred into hypoxic chamber 10\% O2 ((In Vivo2 500; Ruskinn). The duration of the treatment was from 3 to $24 \mathrm{hr}$. Mice had free access to water and food until sacrifice.

\section{Cell cultures}

Primary hepatocytes were isolated from adult WT mice using a collagenase method (Foretz et al., 2010). Hepatocytes were seeded in 6-well plates at a density of 300,000 cells/well and cultured under standard conditions $\left(5 \% \mathrm{CO} 2,37^{\circ} \mathrm{C}\right.$ ) in M199 medium containing $2 \%$ Ultroser $\mathrm{G}$, for $4 \mathrm{~h}$. After cell attachment, the medium was replaced with fresh M199 medium supplemented with $10 \%$ calf serum (Invitrogen).

\section{RT qPCR}

Total RNA was extracted from total duodenum or colon using RNeasy kit (Qiagen). Reverse transcription was performed using iScript Reverse Transcription Supermix for RT-qPCR (BioRad). Realtime PCR was performed on a StepOne cycling machine (Applied Biosystems) with oligos from TaqMan (Life Technologies). Three biological replicates were used for each condition. Data were analyzed with StepOne Plus RT PCR software (v2.1) and Microsoft Excel. Beta-actin transcript levels were used for normalization (delta CT).

Immunohistochemistry

The frozen murine liver sections were obtained using a cryostat (CryoStar NX50) at a thickness of 7 $\mu \mathrm{m}$. Fixation and permeabilization were performed in $4 \%$ paraformaldehyde for $10 \mathrm{~min}$ at room temperature (RT), followed by $0.1 \%$ Triton for 20 min at RT. A 3\% BSA-PBS blocking solution was applied for $1 \mathrm{~h}$ at RT. The primary anti-pSmad1/5/8 antibody (goat polyclonal, Santa Cruz Biotechnology) (1:100) was applied overnight at $4^{\circ} \mathrm{C}$ and resuspended in $1 \%$ BSA-PBS. The secondary antibody (donkey anti-goat), conjugated to Alexa Fluor $568(1: 1,000)$ and resuspended in 1\% BSA-PBS, was added over 30 minutes at RT. Cell nuclei were stained with DAPI $(1: 1,000)$ for $5 \mathrm{~min}$ at RT. Two controls were used in parallel: one with no antibody and the other with only the secondary antibody. Slides were mounted with Fluoromount medium and viewed with the $\times 63$ immersion objective of a confocal microscope (LSM700, Zeiss). Fiji software was used for image processing and measurement of fluorescence intensity per unit area.

\section{Human data}

The 109 healthy controls without any hematological disease were recruited from the orthopedic clinic of Lariboisière University Hospital for 5-HT and hepcidin measurements. Fasting blood samples were collected into vacuum tubes containing $109 \mathrm{mM}$ sodium citrate (Greiner Bio-One) with a 9:1 blood to anticoagulant ratio. After removing $0.5 \mathrm{~mL}$ of whole blood for $5-\mathrm{HT}$ measurement, the remainder was centrifuged at 3,000 $\mathrm{xg}$ for 15 minutes to obtain platelet-poor plasma. The time between blood sampling and plasma isolation was less than 1 hour. Aliquots of whole blood, and plasma were kept frozen $\left(-80^{\circ} \mathrm{C}\right)$ until 5-HT and hepcidin measurements. Whole blood 5-HT was measured by high-pressure liquid chromatography coupled to fluorimetric detection (Kema et al., 1993) and plasma hepcidin by LC-MS/MS (Lefebvre et al., 2015).

Characteristics of the control cohort: age (median, IQR) 38 (29-49); sex M, n (\%) 42(47\%); risk factors ( $\mathrm{n}$, $\%$ ) diabetes $1(1.1 \%)$, arterial hypertension $0(0 \%)$, hyperlipidemia $2(2.2 \%)$, active smoking $3(3.4 \%)$, migraine $1(1.1 \%)$, depression $0(0 \%)$, history of cardiovascular disease $0(0 \%)$, familial history of cardiovascular disease $3(3.4 \%)$, history of autoimmune pathology $1(1.1 \%)$, combined 2 or $>2$ risk factors $2(2.2 \%)$, none $80(90 \%)$. 


\section{References}

Amireault, P., Hatia, S., Bayard, E., Bernex, F., Collet, C., Callebert, J., Launay, J.-M., Hermine, O., Schneider, E., Mallet, J., et al. (2011). Ineffective erythropoiesis with reduced red blood cell survival in serotonin-deficient mice. Proc. Natl. Acad. Sci. U. S. A. 108, 13141-13146.

Amireault, P., Bayard, E., Launay, J.-M., Sibon, D., Le Van Kim, C., Colin, Y., Dy, M., Hermine, O., and Cote, F. (2013). Serotonin is a key factor for mouse red blood cell survival. PloS One 8, e83010.

Anker, S.D., Colet, J.C., Filippatos, G., Willenheimer, R., Dickstein, K., Drexler, H., Lüscher, T.F., Mori, C., von Eisenhart Rothe, B., Pocock, S., et al. (2009). Rationale and design of Ferinject assessment in patients with IRon deficiency and chronic Heart Failure (FAIR-HF) study: a randomized, placebo-controlled study of intravenous iron supplementation in patients with and without anaemia. Eur. J. Heart Fail. 11, 1084-1091.

Choi, W., Namkung, J., Hwang, I., Kim, H., Lim, A., Park, H.J., Lee, H.W., Han, K.-H., Park, S., Jeong, J.S., et al. (2018). Serotonin signals through a gut-liver axis to regulate hepatic steatosis. Nat. Commun. 9, 4824.

Côté, F., Thévenot, E., Fligny, C., Fromes, Y., Darmon, M., Ripoche, M.-A., Bayard, E., Hanoun, N., Saurini, F., Lechat, P., et al. (2003). Disruption of the nonneuronal tph1 gene demonstrates the importance of peripheral serotonin in cardiac function. Proc. Natl. Acad. Sci. U. S. A. 100, 13525-13530.

Ebrahimkhani, M.R., Oakley, F., Murphy, L.B., Mann, J., Moles, A., Perugorria, M.J., Ellis, E., Lakey, A.F., Burt, A.D., Douglass, A., et al. (2011). Stimulating healthy tissue regeneration by targeting the $5-\mathrm{HT}_{2} \mathrm{~B}$ receptor in chronic liver disease. Nat. Med. 17, 1668-1673.

Foretz, M., Hébrard, S., Leclerc, J., Zarrinpashneh, E., Soty, M., Mithieux, G., Sakamoto, K., Andreelli, F., and Viollet, B. (2010). Metformin inhibits hepatic gluconeogenesis in mice independently of the LKB1/AMPK pathway via a decrease in hepatic energy state. J. Clin. Invest. 120, 2355-2369.

Gershon, M.D. (2012). Serotonin is a sword and a shield of the bowel: serotonin plays offense and defense. Trans. Am. Clin. Climatol. Assoc. 123, 268-280; discussion 280.

Haugen, M., Dammen, R., Svejda, B., Gustafsson, B.I., Pfragner, R., Modlin, I., and Kidd, M. (2012). Differential signal pathway activation and 5-HT function: the role of gut enterochromaffin cells as oxygen sensors. Am. J. Physiol. Gastrointest. Liver Physiol. 303, G1164-1173.

Kautz, L., Jung, G., Valore, E.V., Rivella, S., Nemeth, E., and Ganz, T. (2014). Identification of erythroferrone as an erythroid regulator of iron metabolism. Nat. Genet. 46, 678-684.

Kema, I.P., Schellings, A.M., Hoppenbrouwers, C.J., Rutgers, H.M., de Vries, E.G., and Muskiet, F.A. (1993). High performance liquid chromatographic profiling of tryptophan and related indoles in body fluids and tissues of carcinoid patients. Clin. Chim. Acta Int. J. Clin. Chem. 221, 143-158.

Lefebvre, T., Dessendier, N., Houamel, D., Ialy-Radio, N., Kannengiesser, C., Manceau, H., Beaumont, C., Nicolas, G., Gouya, L., Puy, H., et al. (2015). LC-MS/MS method for hepcidin-25 measurement in human and mouse serum: clinical and research implications in iron disorders. Clin. Chem. Lab. Med. 53, 1557-1567.

Long, L., MacLean, M.R., Jeffery, T.K., Morecroft, I., Yang, X., Rudarakanchana, N., Southwood, M., James, V., Trembath, R.C., and Morrell, N.W. (2006). Serotonin increases susceptibility to pulmonary hypertension in BMPR2-deficient mice. Circ. Res. 98, 818-827.

Meynard, D., Kautz, L., Darnaud, V., Canonne-Hergaux, F., Coppin, H., and Roth, M.-P. (2009). Lack of the bone morphogenetic protein BMP6 induces massive iron overload. Nat. Genet. 41, 478-481. 
Nemeth, E., Tuttle, M.S., Powelson, J., Vaughn, M.B., Donovan, A., Ward, D.M., Ganz, T., and Kaplan, J. (2004). Hepcidin regulates cellular iron efflux by binding to ferroportin and inducing its internalization.

Science 306, 2090-2093.

Pocock, R., and Hobert, O. (2010). Hypoxia activates a latent circuit for processing gustatory information in C. elegans. Nat. Neurosci. 13, 610-614.

Roth, M.-P., Meynard, D., and Coppin, H. (2019). Regulators of hepcidin expression. Vitam. Horm. 110, 101-129.

Shepherd, A.P. (1982). Metabolic control of intestinal oxygenation and blood flow. Fed. Proc. 41, 20842089.

Sibon, D., Coman, T., Rossignol, J., Lamarque, M., Kosmider, O., Bayard, E., Fouquet, G., Rignault, R., Topçu, S., Bonneau, P., et al. (2019). Enhanced Renewal of Erythroid Progenitors in Myelodysplastic Anemia by Peripheral Serotonin. Cell Rep. 26, 3246-3256.e4.

Taylor, C.T., and Colgan, S.P. (2007). Hypoxia and gastrointestinal disease. J. Mol. Med. Berl. Ger. 85, $1295-1300$.

Van Aelst, L.N.L., Abraham, M., Sadoune, M., Lefebvre, T., Manivet, P., Logeart, D., Launay, J.-M., Karim, Z., Puy, H., and Cohen-Solal, A. (2017). Iron status and inflammatory biomarkers in patients with acutely decompensated heart failure: early in-hospital phase and 30-day follow-up. Eur. J. Heart Fail. 19, 10751076 .

Walther, D.J., Peter, J.-U., Bashammakh, S., Hörtnagl, H., Voits, M., Fink, H., and Bader, M. (2003). Synthesis of serotonin by a second tryptophan hydroxylase isoform. Science 299, 76.

Young, R.L., Lumsden, A.L., Martin, A.M., Schober, G., Pezos, N., Thazhath, S.S., Isaacs, N.J., Cvijanovic, N., Sun, E.W.L., Wu, T., et al. (2018). Augmented capacity for peripheral serotonin release in human obesity. Int. J. Obes. 2005 42, 1880-1889. 